\title{
Ensuring all falling trees are heard: the importance of publishing all research
}

\author{
Hilary P. Grocott, MD, FRCPC, FASE • Philip M. Jones, MD, FRCPC
}

Received: 19 March 2018/Revised: 26 March 2018/Accepted: 26 March 2018/Published online: 27 April 2018

(c) Canadian Anesthesiologists' Society 2018

"If a tree falls in the forest and no one is there to hear it, does it make a sound?”

In this issue of the Journal, McKay et al. report the results of a randomized-controlled trial (RCT) of 200 patients in whom an $\mathrm{LMA}^{\circledR}$ Classic $^{\mathrm{TM}}$ (Teleflex Inc.; Morrisville, NC, USA) supraglottic airway (SGA) was inserted. ${ }^{1}$ The incidence of sore throat was compared in groups using lidocaine- or water soluble-based lubricants and no lubricant at all. Their results suggest that lubrication does not influence the occurrence of sore throat following insertion of the LMA Classic. Though the results of this study are not particularly earth-shattering, and are also somewhat dated (making its relevance somewhat uncertain with respect to the newer generation SGA devices that are now being used), what is particularly notable is when (i.e., what year) this study was undertaken. Indeed, this RCT commenced almost 15 years ago and finished 11 years ago, yet its reporting was delayed until 2018.

Astute readers will also note that this trial was not registered on a publicly available website, as is now a requirement for the Journal (and most other reputable publishing platforms). This is because the work pre-dated the required use of these registration sites. Nevertheless, McKay et al. circumvent some of these

H. P. Grocott, MD, FRCPC, FASE ( $₫)$

Department of Anesthesiology, Perioperative \& Pain Medicine,

St. Boniface Hospital, University of Manitoba, CR3008 - 369

Tache Avenue, Winnipeg, MB R2H 2A6, Canada

e-mail: hgrocott@sbgh.mb.ca

P. M. Jones, MD, FRCPC

Departments of Anesthesia \& Perioperative Medicine and Epidemiology \& Biostatistics, University of Western Ontario, London, ON, Canada pitfalls associated with the failure to register by making their Research Ethics Board approval and original study protocol available (as Electronic Supplementary Material) so there would be no questions raised as to what the primary endpoint and analytical plan might have been (or changed to). ${ }^{2}$ We are pleased that Dr. McKay chose to persevere and report these data, despite the lengthy delay from their acquisition. The author's stated reasons for this "closure" include an impending personal retirement and the wish to "clear the desk" with respect to any unpublished research data (personal communication, William McKay). This is a laudable act and an important lesson for other investigators.

Publication of these results, late though they may be, highlights an important and broader issue in medical research. Estimates vary, but even with the most rigorous studies, the failure to publish is approximately $30 \% .^{3}$ There are a multitude of reasons why the results of RCTs are not reported, ranging from investigators being distracted by newer, more contemporary research directions, to investigator illness or retirement. More worrisome are purposeful delays resulting from a desire of investigators or sponsors to distract clinicians from null treatment effects or adverse effects exposed by the trials. When one adds this to the ever present issue of publication bias (i.e., the likelihood that positive trials are published more often than negative trials) ${ }^{4,5}$ present in the anesthesia (and most of the other medical) literature, this risk of a systemic distortion of the literature easily becomes apparent. Some delays are entirely innocent, legitimate, and related to additive delays in peer review; e.g., investigators "surf" from one journal to another, looking for acceptance of their scholarly work.

There are many reasons why the results of all RCTs (be they incomplete, outdated, and/or negative) should always be reported. The most important is that there is an implicit 
ethical contract that the investigator enters with the patient during the consent process that requires that these patients' efforts, and the risks that they agree to undertake when involved in the trial, inform the broader subject area by having their data available. This is similarly implicit in the Declaration of Helsinki regarding the ethical principles for medical research in humans. ${ }^{6}$ In addition, any data collected, even if "negative", can help inform further systematic reviews on the subjects. This is particularly true when individual patient-level data are made freely available by researchers. ${ }^{7}$ Patient-level data (in contrast to study-level data taken from published tables) improve the precision of systematic reviews by permitting researchers to condition effect estimates on patient-level characteristics, such as demographic variables or comorbidities. The importance of publishing all results from all trials is a focus of several organizations including those online (http://www.alltrials.net) and on social media (@FDAAAtracker).

In summary, although the actual results of this particular trial may not neccearily change practice, what is noteworthy is the tremendous efforts that have been made to ensure that the ethical contract with these patients, and the funding sources that contributed to the ability to undertake this trial, is rewarded with the results being published. Its publication serves as a contract fulfilled and a career objective no doubt similarly fulfilled. Just as a tree falling in the forest needs someone there to hear it, research must be published for the participant's contribution to be heard.

\section{S'assurer d'entendre tous les arbres tomber : l'importance de publier toutes les recherches}

\section{"Si un arbre tombe dans la forêt et que personne ne l'entend, fait-il du bruit?»}

Dans ce numéro du Journal, McKay et coll. présentent les résultats d'un essai randomisé contrôlé (ERC) avec 200 patients chez qui un masque laryngé (un dispositif de voies respiratoires supraglottiques SGA) LMA ${ }^{\circledR}$ classic $^{\mathrm{MD}}$ (Teleflex Inc.; Morrisville, NC, États-Unis) a été inséré. ${ }^{1}$ L'incidence du mal de gorge a été comparée entre 3 groupes après l'utilisation d'un lubrifiant à base de lidocaïne ou d'un lubrifiant hydrosoluble, ou sans utilisation de lubrifiant. Leurs résultats portent à croire que la lubrification n'a pas d'influence sur la survenue d'un mal de gorge après l'insertion du LMA Classic. Bien que les résultats de cette étude ne soient pas particulièrement renversants et qu'ils soient anciens (rendant leur pertinence plutôt incertaine face à la nouvelle génération de dispositifs SGA maintenant utilisés) ce qui est particulièrement intéressant est de savoir quand (c'est-à-dire en quelle année) cette étude a été entreprise. Cet ECR a en effet commencé il y a presque 15 ans et a été clos il y a 11 ans, or sa publication a été retardée jusqu'en 2018.

Les lecteurs perspicaces auront également noté que cet essai n'a pas été enregistré sur un site Web publiquement disponible, comme cela est maintenant obligatoire pour le Journal (et pour la majorité des autres plateformes de publication fiables). Cela est dû au fait que ce travail était antérieur à l'obligation d'utilisation de ces sites d'enregistrement. Néanmoins, McKay et coll. ont contourné ces écueils associés à l'absence d'enregistrement en rendant publique l'approbation de leur Comité d'éthique de la recherche et le protocole original de l'étude (sous forme de documents électroniques supplémentaires) de façon à ce qu'aucune question ne soit soulevée concernant le critère d'évaluation principal, le plan analytique ou de leur changement. ${ }^{2}$ Nous sommes heureux que le Dr McKay ait choisi de persévérer et de publier ces données en dépit du long délai écoulé depuis leur acquisition. Les motifs de cette publication tardive fournis par les auteurs incluent la retraite imminente de l'un d'entre eux et le désir de finaliser le chapitre des données de recherche non publiées (communication personnelle, William McKay). Il s'agit d'un geste louable et d'une leçon importante pour d'autres investigateurs.

La publication de ces résultats, aussi tardifs soient-ils, met en évidence un problème plus important et plus large dans la recherche médicale. Les estimations varient, mais même pour les études les plus rigoureuses, la nonpublication est d'environ $30 \%{ }^{3}$ Une multitude de raisons est invoquée pour expliquer pourquoi les résultats d'ECR ne sont pas publiés, allant d'investigateurs se lançant dans des sujets de recherches plus contemporains jusqu'à la maladie ou la retraite de l'investigateur principal. Plus troublants sont les retards volontaires résultant du désir des investigateurs ou promoteurs de détourner les cliniciens d'un effet thérapeutique nul, voire d'effets indésirables mis en évidence par les études. Quand on ajoute cela au problème toujours présent des biais de publications (c.-à-d. la probabilité que des essais positifs soient publiés plus souvent que des essais négatifs) ${ }^{4,5}$ présents en anesthésie et dans la majorité des publications médicales, le risque de distorsion systémique de la littérature n'en devient que trop facilement évident. Quelques retards sont parfaitement innocents, légitimes et liés aux retards cumulatifs de l'examen par les pairs quand, par exemple, les investigateurs changent de journaux, afin d'obtenir l'acceptation de leur travail. 
Il y a de nombreuses raisons pour lesquelles les résultats de tous les ERC (qu'ils soient incomplets, dépassés et/ou négatifs) devraient toujours être publiés. Le plus important est qu'il existe un contrat éthique implicite passé entre l'investigateur et le patient au moment du processus de consentement éclairé qui exige que les efforts de ces patients - et les risques qu'ils acceptent de prendre en s'engageant dans l'étude - servent le domaine d'étude en rendant leurs données disponibles. Cela est similairement implicite dans la Déclaration d'Helsinki concernant les principes éthiques pour la recherche médicale chez les humains. ${ }^{6}$ De plus, toutes les données recueillies, même « négatives » contribuent à renseigner davantage les revues systématiques dans les domaines concernés. Cela est particulièrement vrai quand des données au niveau de chaque patient sont mises librement à la disposition des chercheurs. $^{7}$ Les données au niveau des patients (par opposition aux données au niveau de l'étude provenant des tableaux publiés) améliorent la précision des revues systématiques en permettant aux chercheurs de lier conditionnellement l'estimation des effets aux caractéristiques de chaque patient, telles que les variables démographiques ou les comorbidités. L'importance de la publication de tous les résultats de tous les essais est un objectif de plusieurs organisations, y compris d'organisations sur le Web (http://www.alltrials.net) et sur les réseaux sociaux (@FDAAAtracker).

En résumé, bien que les véritables résultats de cet essai particulier ne vont pas nécessairement modifier les pratiques, il faut noter que d'énormes efforts ont été accomplis pour s'assurer que le contrat éthique passé avec ces patients et les sources de financement ayant contribué à la réalisation de cet essai est rempli avec la publication des résultats. Sa publication représente le terme d'un contrat rempli et d'un objectif de carrière sans aucun doute également atteint. Tout comme un arbre tombant dans la forêt a besoin que quelqu'un l'entende, la recherche doit être publiée pour que la contribution des participants soit entendue.

Conflicts of interest None declared.

Editorial responsibility This submission was handled by Dr. Gregory L. Bryson, Deputy Editor-in-Chief, Canadian Journal of Anesthesia.

Conflits d'intérêts Aucun déclaré.

Responsabilité éditoriale Cet article a été traité par le $\mathrm{D}^{\mathrm{r}}$ Gregory L. Bryson, rédacteur en chef adjoint, Journal canadien d'anesthésie.

\section{References}

1. McKay WP, Maslany J, Schubert EP. A randomized trial of three lubrication strategies on sore throat after insertion of the LMA ${ }^{\circledR}$ Classic $^{\mathrm{TM}}$ supraglottic airway. Can J Anesth 2018; 65: DOI: https://doi.org/10.1007/s12630-018-1132-0.

2. Jones PM, Chow JT, Arango MF, et al. Comparison of registered and reported outcomes in randomized clinical trials published in anesthesiology journals. Anesth Analg 2017; 125: 1292-300.

3. Jones CW, Handler L, Crowell KE, Keil LG, Weaver MA, PlattsMills $T F$. Non-publication of large randomized clinical trials: cross sectional analysis. BMJ 2013; 347: f6104.

4. Chong SW, Collins NF, Wu CY, Liskaser GM, Peyton PJ. The relationship between study findings and publication outcome in anesthesia research: a retrospective observational study examining publication bias. Can J Anesth 2016; 63: 682-90.

5. Jones $P M$. Publication bias in the anesthesiology literature: shifting the focus from the "positive" to the "truth". Can J Anesth 2016; 63: 658-63.

6. World Medical Association. WMA Declaration of Helsinki Ethical Principles for Medical Research Involving Human Subjects. Available from URL: www.wma.net/policies-post/ wma-declaration-of-helsinki-ethical-principles-for-medicalresearch-involving-human-subjects/ (accessed March 2018).

7. Jones PM, Bryson GL, Backman SB, Mehta S, Grocott HP. Statistical reporting and table construction guidelines for publication in the Canadian Journal of Anesthesia. Can J Anesth 2018; 65: 152-61. 